\title{
BIOPSIEEINRICHTUNG ZUR HISTOLOGISCHEN SICHERUNG VON KLEINSTTUMOREN IN DER MR-MAMMOGRAPHIE
}

\author{
O. Wendt ${ }^{1}$, J. Oellinger ${ }^{2}$, U. Boenick ${ }^{1}$, R. Felix ${ }^{2}$, T.C. Lüth ${ }^{3}$, D. Schauer ${ }^{2}$ \\ ${ }^{1}$ Technische Universität Berlin, Institut für Mikrotechnik und Medizintechnik \\ ${ }^{2}$ Universitätsklinikum Charité Berlin, Campus Virchow-Klinikum, Strahlen- und Poliklinik \\ ${ }^{3}$ Universitätsklinikum Charité Berlin, Campus Virchow-Klinikum, Klinik für Mund-, Kiefer- und \\ Gesichtschirurgie
}

owendt@bmt1.kf.tu-berlin.de

\section{EINLEITUNG}

Die kontrastmittelunterstützte Magnetresonanztomographie der Mamma (MR-Mammographie) hat sich in den vergangenen Jahren bei gezielter Indikationsstellung zunehmend in der Mammadiagnostik etabliert. Dabei findet man im Einzelfall suspekte Herdbefunde, die weder palpatorisch, mammographisch noch sonographisch nachgewiesen werden können. Der wesentliche Vorteil dieses Verfahrens besteht in der sehr hohen Sensitivität im Nachweis des invasiv wachsenden Mammakarzinoms. Die wichtigste Voraussetzung für die erfolgreiche Durchführung einer bildbasierten Biopsie ist die sichere räumliche Punktion des Tumors. Dies ist nur im unmittelbaren Anschluß an die Mammographie bei sicherer Fixierung der Mamma zu erreichen. Die erschwerten räumlichen Bedingungen im MRT erfordern eine externe Punktion des Tumors. Die bei herkömmlichen Systemen notwendige Patientenverlagerung sowie eine ungenügende Erfolgskontrolle durch Überprüfung der Lage der Punktionsnadel führen zu erheblichen Limitierungen des Biopsieerfolges.

\section{KONZEPTION UND METHODIK}

Die Biopsieeinrichtung ist auf der Patientenliege befestigt und die Patientin liegt in Bauchlage auf der Liegefläche der Oberseite des Rahmengestells. Einrichtung und Patientin befinden sich im Isozentrum des Magnetresonanztomographen, so daß eine simultane Untersuchung und Behandlung beider Mammae der Patientin von cordaler als auch cranialer Richtung möglich sind. Die Fixierung der zu untersuchenden Strukturen, die Bildakquisition, die Bildauswertung sowie diagnostische und therapeutische Schritte werden in dieser ursprünglich eingenommenen Patienten-position innerhalb des Tomographen durchgeführt (s. Abb.1). Fixierung, Instrumente und Navigationssystem sind in die Biopsieeinrichtung integriert. Die Empfangsspule ist in der Oberseite des Rahmengestells befestigt. Das System ist in einem geschlossenen Magnetresonanztomographen nutzbar, wodurch sich die Möglichkeit der Anwendung in offenen oder halboffenen Geräten ohne weiteres ergibt.

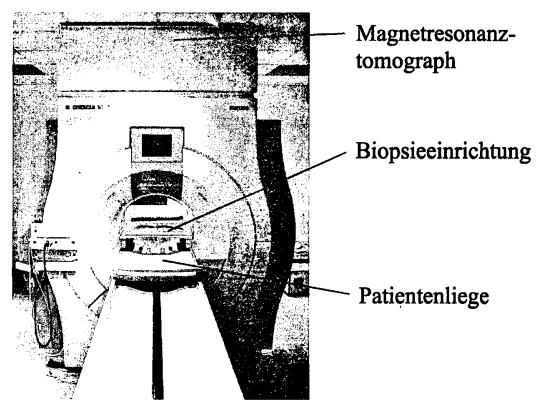

Abbildung 1: MRT mit Biopsieeinrichtung

Alle aktiven und passiven Komponenten der Biopsieeinrichtung sind werkstofftechnisch in singulärer und kombinierter Form so ausgelegt, daß ein Entstehen von Bildartefakten oder Deflektionen ausgeschlossen werden kann. So zeigen Untersuchungen der Effekte piezoelektrischer Motoren in starken Magnetfeldern eine klare Indikation für deren Anwendbarkeit als Antriebselement [1].

\section{AUFBAU UND WIRKUNGSWEISE}

Das Fixationssystem gewährleistet eine hohe Ortsauflösung der Punktierbarkeit des Ziellumens bei gleichzeitig individueller Wahl der Kompressionskraft (s. Abb. 2). Es besteht aus jeweils zwei Platten, die mit Löchern versehen sind, welche aufgrund ihrer Geometrie, d.h. einer kugelförmigen Aussparung in der Instrumentenaufnahme und einer konischen Aussparung auf der gegenüberliegenden Seite, ein Kippen der Instrumentenführung gleichzeitig in longitudinaler sowie azimutaler Richtung erlauben. In die Platten sind mit Kontrastmittel gefülte Marker integriert, welche im Bildbereich identifiziert werden. In dem so erzeugten Koordinatensystem wird die suspekte Läsion lokalisiert.

Das Navigationssystem ist in transversaler, coronarer und sagitaler Ebene linear zu bewegen. Durch formschlüssige Kugel-Pfanne-Verbindung des Instrumentennavigationssystems mit dem Fixationssystem als 
Rotationszentrum kann, bei gekoppelter linearer Bewegung des Navigationssystems und kontinuierlicher Positionsanpassung, eine longitudinale als auch azimutale Drehung und somit eine freie Wahl des Inzisionswinkels des Instrumentes erfolgen.

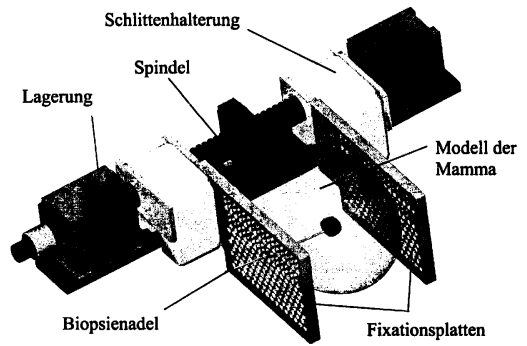

Abbildung 2: Fixationssystem

Die Gewebeentnahme erfolgt als Tru-cut-Biopsie, unter Anwendung eines für diesen speziellen Anwendungsfall entwickelten Instrumentes. Dieses ist nichtmanuell steuerbar und für einen Einsatz innerhalb des Isozentrums von Kernspintomographen geeignet. Die Biopsienadel besteht aus einem stilettförmigen Mandrin, an dessen distalem Ende eine seitliche Aufnahme der Probe eingebracht ist, und einer Kanüle, in der das Mandrin geführt wird. Diese sind in einem Mandrinsowie einem Kanülenhalter fixiert und ineinander koaxial verschiebbar. Die Bewegung des Mandrins und der Kanüle sowie das Lösen von Mandrin- und Kanülenhalter aus einer Fixierung erfolgen ohne manuelles Einwirken, unter Anwendung elektrischer Aktuatoren. In Abbildung 3 ist das Navigationssystem mit dem Biopsieinstrument dargestellt.

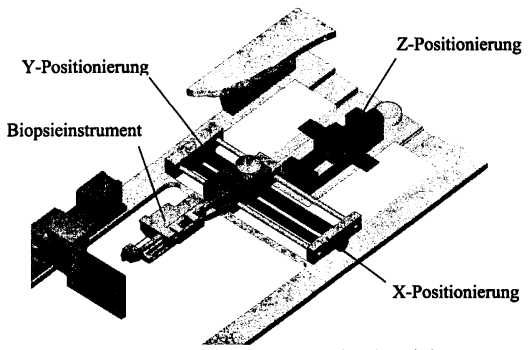

Abbildung 3: Navigationssystem mit Biopsieinstrument

Die Lokalisation der zu untersuchenden Läsion sowie die Berechnung der Zielkoordinaten der Punktion erfolgt unter Nutzung einer Biopsie-Software. Diese liest Bilddaten im DICOM3.0-Format, konvertiert die Informationen bezüglich Bild-, Sequenz- und Patientendaten und gewährleistet eine flexible Bewegung zwischen den aufgenommenen Schichten. Durch die Transformation der Sequenzinformationen (Schichtdicke, - anzahl, Ausrichtung u.a.) in das Koordinatensystem des Fixationssystems werden die Zielkoordinaten der Läsion berechnet.

Die spektroskopischen Eigenschaften und die Bildwiedergabe der Empfängerspule (Doppel-Brust-Spule) sind optimiert [2].

Der invasiv tătige Radiologe führt wichtige Untersuchungsschritte, wie z.B. die Fixierung der Mammae, die Inzision oder das Auslösen der Biopsie, in unmittelbarer Nähe der Patientin manuell durch. Die Ausrichtung und Bewegung der Instrumente erfolgt automatisch.

\section{ERGEBNISSE}

Die Biopsieeinrichtung gewährleistet eine Punktion suspekter Läsionen geringer Ausdehnung (Durchmesser kleiner $3 \mathrm{~mm}$ ). Dies ist auf die automatische Steuerung der Einrichtung sowie auf die Kompensation der Bewegung der Patientin aus dem MRT zurückzufuhren.

Durch die Verwendung dia- und paramagnetischer Materialien in der Konstruktion sowie nichtmagnetischer Antriebs- und Steuerungselemente sind die Einflüsse von Artefakten- und Deflektionen minimal.

\section{DISKUSSION UND AUSBLICK}

Durch die Realisierung einer Biopsieeinrichtung zur Anwendung innerhalb des Isozentrums von Magnetresonanztomographen können sowohl die MRMammographie als auch die histologische Sicherung von Gewebeproben mit einer hohen Spezifizität und Punktionsgenauigkeit durchgeführt werden. Die physische sowie psychische Belastung der Patientin sind durch den geringeren zeitlichen Aufwand, die hohe Treffsicherheit sowie die kontinuierliche Kontrolle der Untersuchung im Bildbereich gering.

Das Ziel weiterer Entwicklungen ist die Gewährleistung einer schnellen histopathologischen Beurteilung der Probe sowie die Realisierung eines therapeutischen Moduls zur Behandlung der Läsion, z.B. mittels thermischer Applikatoren (Kryo- oder Lasertechnik).

\section{LITERATUR}

[1] O. Wendt, J. Oellinger, T.C. Lüth, R. Felix, U. Boenick, "The Effects of the Use of Piezoelectric Motors in a 1.5-Tesla High-Field Magnetic Resonance Imaging System" in Biomedizinische Technik Band 45, Heft 1-2/2000, S. $20-25$

[2] M. Hentschel, J. Oellinger, C. Siewert, H. Wieder, H. Hosten, O. Wendt, T.C. Lüth, U. Boenick, R. Felix, " ${ }^{11}$ and ${ }^{31} \mathrm{P}$ NMR Characterisation of a Double Breast Coil for Spectroscopic Measurements and Imaging" in Biomedizinische Technik Band 44, Heft $10 / 1999$, S. 272-277 